\title{
Different Contributions of the Hippocampus and Perirhinal Cortex to Recognition Memory
}

\author{
Huimin Wan, ${ }^{1}$ John P. Aggleton, ${ }^{2}$ and Malcolm W. Brown ${ }^{1}$ \\ ${ }^{1}$ Department of Anatomy, University of Bristol, Medical School, Bristol, BS8 1TD, United Kingdom, and 2School of \\ Psychology, University of Wales, College of Cardiff, Cardiff, CF1 3YG, United Kingdom
}

\begin{abstract}
Brain regions involved in visual recognition memory, including the hippocampus, have been investigated by measuring differential neuronal activation produced by novel and familiar pictures. Novel and familiar pictures were presented simultaneously, one to each eye, using a paired viewing procedure. Differential neuronal activation was determined using immunohistochemistry for the protein products of c-fos as an imaging technique. The results establish that the regions of the rat brain associated with discriminating the novelty or familiarity of an individual item (such as a single object) differ from those responding to a spatial array of items (such as a scene). Perirhinal cortex and area TE of the temporal lobe are activated significantly more by pictures of novel than of familiar individual objects, but the hippocampus is not differentially activated. In
\end{abstract}

There has been much recent interest in defining those brain regions responsible for recognition memory and, in particular, uncovering the involvement of the hippocampus. The results of electrophysiological and recent lesion experiments have thrown doubt on the importance of the hippocampus for the performance of tasks such as delayed matching to sample that are soluble by judging the relative familiarity or recency of occurrence of discrete, individual stimulus items while emphasizing the crucial role of perirhinal cortex in such tasks (Brown, 1996; Murray, 1996; Suzuki, 1996; Brown and Xiang, 1998; Xiang and Brown, 1998; Aggleton and Brown, 1999).

More generally, recognition memory requires discrimination of the novelty or familiarity of previous experiences. These experiences may be of an individual item such as an object or of a complete environment or scene made up of a spatial arrangement of many items. Thus for example, we immediately recognize that someone has placed a new chair in a room of our home. Equally, we immediately recognize that someone has made a new arrangement of the familiar items of furniture in a room of our home. To model these situations and thereby assess whether the same brain regions are involved in judging the relative familiarity of individual items as for arrangements of items, rats were exposed to pictures of such stimuli. In the first experiment, the novel and familiar pictures were of individual stimulus items; in the second

Received Aug. 6, 1998; revised Nov. 16, 1998; accepted Nov. 19, 1998.

This work was supported by the Medical Research Council. We thank Dr. D. Hancock, Biochemistry of the Cell Nucleus Laboratory, Imperial Cancer Research Institute for supplying the primary antibody, and L. Ni, A. Griffiths, and J. Leendertz for technical assistance.

Correspondence should be addressed to Dr. M. W. Brown, Department of Anatomy, University of Bristol, Medical School, Bristol, BS8 1TD, UK.

Copyright (C) 1999 Society for Neuroscience $0270-6474 / 99 / 191142-07 \$ 05.00 / 0$ contrast, pictures of novel arrangements of familiar items produce significantly greater activation than familiar arrangements of these items in postrhinal cortex and subfield CA1 of the hippocampus but significantly less activation in the dentate gyrus and subiculum; perirhinal cortex and area TE are not differentially activated. Thus, the hippocampus is importantly involved in processing information essential to recognition memory concerning the relative familiarity of arrangements of items, as needed for episodic memory of scenes, whereas the perirhinal cortex processes such information for individual items.

Key words: Fos; immunoreactivity; rat; recognition memory; hippocampus; rhinal cortex

experiment, the pictures were of novel or familiar arrangements of familiar items.

The activation produced by these pictures in different regions of the intact brain was imaged using immunohistochemistry for the protein products (Fos) of the immediate early gene c-fos (Sheng and Greenberg, 1990; Morgan and Curran, 1991; Dragunow, 1996; Larea, 1997). This technique makes possible the simultaneous examination of the activity of populations of neurons in multiple brain regions. However, the results of such imaging are only easily interpretable if the behavioral and stimulus presentation conditions are closely controlled. For this reason, in the present experiments each rat was trained to hold its head in an observing hole while pictures were displayed in front of it on two computer monitors using a paired viewing procedure (Zhu et al., 1996). In this procedure a rat sees simultaneously a familiar picture with one eye and a novel picture with the other eye (Fig. 1). The procedure thus ensures that both pictures are seen under the same conditions of alertness and with similar eye movements. The two pictures are spaced apart to each side of the rat so that one stimulus is viewed by the monocular visual field of the right eye while the other was similarly viewed by the left eye. Information from each eye initially passes chiefly to the contralateral cerebral hemisphere (Sefton and Dreher, 1995).

\section{MATERIALS AND METHODS}

Subjects and apparatus. Subjects were 16 male pigmented rats (DA strain; Bantin and Kingman, Hull, UK), weighing from 175 to $220 \mathrm{gm}$. All animal procedures were performed in accordance with United Kingdom Home Office Licensing Laws. Each of the two experiments (with pictures of individual items or arrangements of items) used eight rats. Each rat was trained in a viewing chamber $(30 \times 30 \times 35 \mathrm{~cm})$. The top of the chamber was open, the bottom and sides were black; the front was transparent (Perspex) with a central observing hole $3 \mathrm{~cm}$ in diameter, 6 $\mathrm{cm}$ above the floor. In the chamber, $4.5 \mathrm{~cm}$ to either side of the observing 


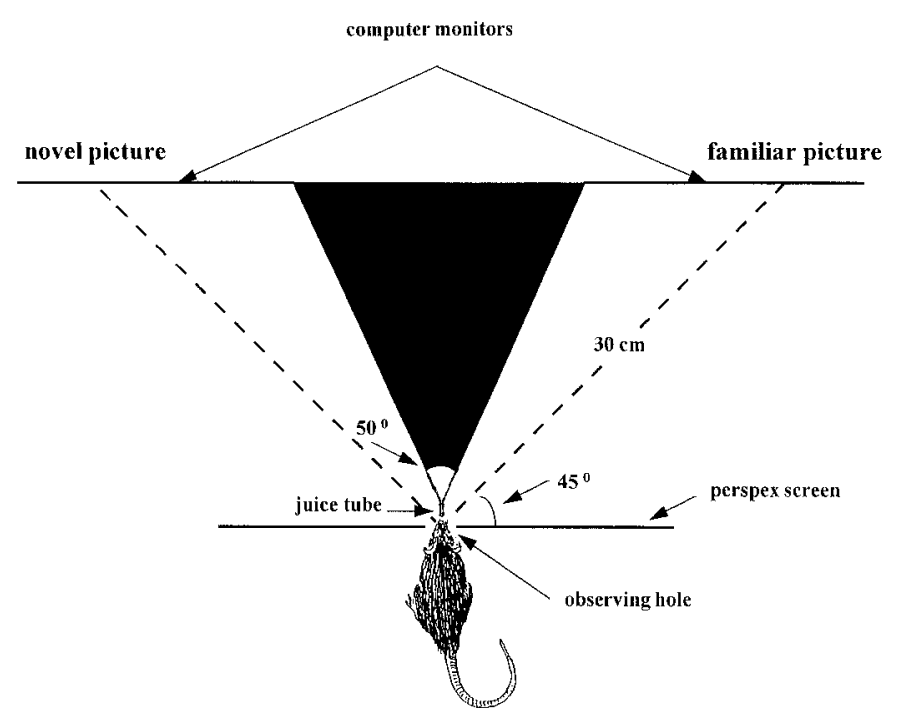

Figure 1. Apparatus for the paired viewing procedure. A novel and a familiar picture appeared simultaneously, one on each of the two computer monitors, when the rat's head was in the observing hole. The two monitors were spaced apart to each side of the rat so that one was viewed by the monocular visual field of the right eye while the other was similarly viewed by the left eye. When the rat's head was in the observing hole, the partition prevented the right eye seeing the left monitor and vice versa.

hole, ran two small barriers $(12 \mathrm{~cm}$ long $\times 9 \mathrm{~cm}$ high); these kept the rat's body at $90^{\circ}$ to the front screen when its head was in the observing hole. When the rat's head was positioned in the observing hole, an infrared beam was interrupted, signaling the computer (Viglen Pentium PC) to start a trial. After a variable interval of 3-4 sec, provided the head remained in the hole, two pictures (each $15 \times 12 \mathrm{~cm}$ ) were shown simultaneously for $4.5 \mathrm{sec}$, one on each of the two computer monitors placed $30 \mathrm{~cm}$ from the observing hole ("paired viewing procedure"; Fig. 1). A black partition ensured that the rat's left eye could not see the right monitor screen, and his right eye could not see the left screen. After the pictures had been shown for $4 \mathrm{sec}$ a drop of diluted blackcurrant juice was delivered by a metal tube that the rat could just reach and lick. No other behavioral contingency was required, and the procedure ensured that the rat's behavior (which was monitored by camera and videorecorded) was the same for the novel and familiar pictures.

Stimuli. In the first experiment, each picture was of a different individual item (chosen from MicroSoft Clipart). In the second experiment, each picture was of a different arrangement of items, each arrangement being constructed from three individual items. The arrangements comprised six sets of three individual items in ten different spatial arrangements (five familiar, five novel). Novel and familiar arrangements used the same spatial locations on the computer screen, but with different members of the set in the particular locations.

Behavioral procedure. From 1 week before training started, each rat was kept on a $12 \mathrm{hr}$ dark/light cycle, with the dark phase being during daylight. During training the rats were allowed ad libitum access to water for $2 \mathrm{hr}$ each day. Each rat was pretrained for $2 \mathrm{~d}$, without stimulus presentation, to go to the observing hole for juice reward. The subsequent training period lasted $6 \mathrm{~d}$, with two morning sessions and one afternoon session each day. The second morning session followed the first without a break; the afternoon session was $3 \mathrm{hr}$ later. In each session two sets of 30 different pictures were presented, one set on each monitor, a picture from each set being shown simultaneously on each of the 30 trials. In both morning sessions the same two sets of pictures were used. In each afternoon session one eye was exposed to a set of novel pictures while the other eye saw a particular one of the two sets of pictures presented in the morning (the "familiar set"). A different novel set of stimuli was shown each afternoon to familiarize the animal with seeing novel and familiar stimuli simultaneously. The different sets of novel and familiar stimuli were presented so that by the end of the experiment each eye had seen the same number of novel and familiar sets of stimuli, and both eyes had seen the familiar set the same number of times; the familiar set had then been seen 18 times ( 9 times by each eye). The "novel set" of

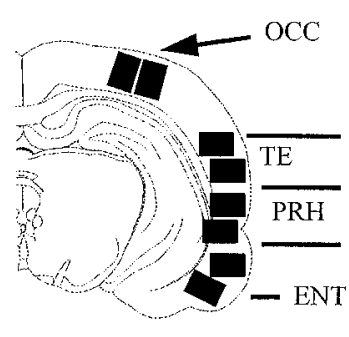

$-5.20 \mathrm{~mm}$

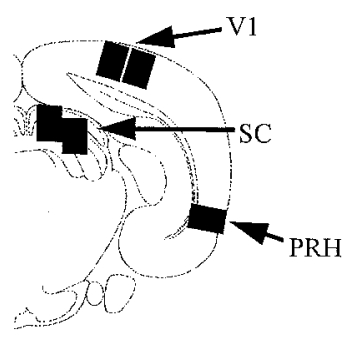

$-6.72 \mathrm{~mm}$

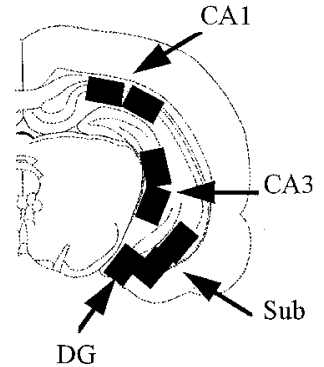

$-5.20 \mathrm{~mm}$

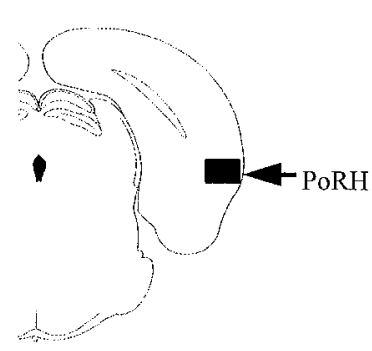

$-7.80 \mathrm{~mm}$
Figure 2. Diagrams of coronal sections indicating areas sampled. The distance (in millimeters) of the sections from bregma is indicated (Paxinos and Watson, 1986). So that processing of the different areas could be done in parallel, only the illustrated brain levels were sampled. One area was sampled for the dentate gyrus and postrhinal cortex, three areas for perirhinal cortex, and two for all other regions. The significance or nonsignificance of any of the reported effects was not dependent on these differences in the number of sampling points. $C A 1, C A 3$, Hippocampal subfields; $D G$, dentate gyrus; $E N T$, entorhinal cortex; $O C C$, occipital cortex; PoRH, postrhinal cortex; $P R H$, perirhinal cortex; $S C$, superior colliculus; $T E$, area TE of the inferotemporal cortex; $V 1$, primary visual cortex; $V L G$, ventral lateral geniculate nucleus of the thalamus. The locations of PRH and PoRH are modeled after Burwell et al. (1995).

stimuli was shown with the familiar set on the last afternoon, $1.5 \mathrm{hr}$ before the animal was anesthetized deeply with pentobarbitone and perfused with $0.1 \mathrm{M}$ phosphate buffer and $4 \%$ paraformaldehyde, $\mathrm{pH} 7.4$. The familiar set for one animal became the novel set for the next so that stimulus materials were counterbalanced across rats. Further, the eye (left or right) that viewed the novel set was also counterbalanced across animals.

Tissue-processing procedure. After perfusion, the brain was removed and placed for $12 \mathrm{hr}$ in $4 \%$ paraformaldehyde followed by $24 \mathrm{hr}$ in $30 \%$ sucrose. The immunohistochemical and counting procedures followed those of Zhu et al. (1995). Briefly, coronal sections $(25 \mu \mathrm{m})$ were cut on a cryostat, and floating sections were processed using a primary antibody and the avidin-biotin complex (ABC, Vector Laboratories, Burlingame, CA) method. The primary antibody, kindly provided by Dr. D. Hancock (Biochemistry of the Cell Nucleus Laboratory, Imperial Cancer Research Institute), was a rabbit polyclonal directed against the $\mathrm{N}$-terminal region of rat c-fos peptide and is c-fos-specific (Brennan et al., 1992). The secondary antibody was biotinylated goat anti-rabbit (Vector Laboratories). DAB was used for visualization of Fos immunoreactivity, and the automated counting of stained nuclei was performed using an image analysis system (SeeScan Ltd.) (McCabe and Horn, 1994; Zhu et al., $1995 b)$. Processing and counting were done without the experimenter knowing which eye had seen the novel set. Counts above threshold were obtained from the right and left hemispheres for rectangular areas $(0.94 \times 0.67 \mathrm{~mm})$ from two sections for each brain region (Fig. 2). For data analysis, each count was first normalized by dividing it by the corresponding mean for the particular area averaged across both hemispheres for the particular rat. The normalized counts were then subjected to an ANOVA with factors: rat, area, and novelty/familiarity. Statistical tests (two-tailed) used a significance level of 0.05 . 
a.

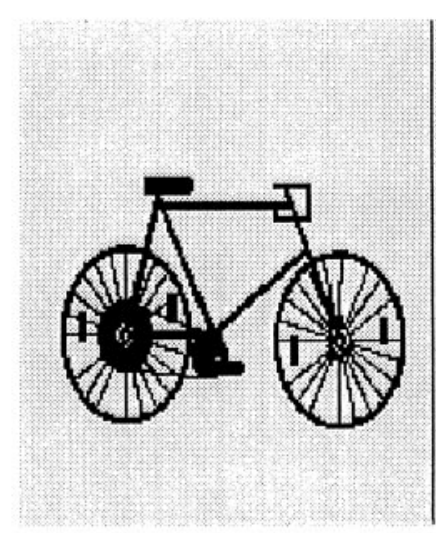

b.

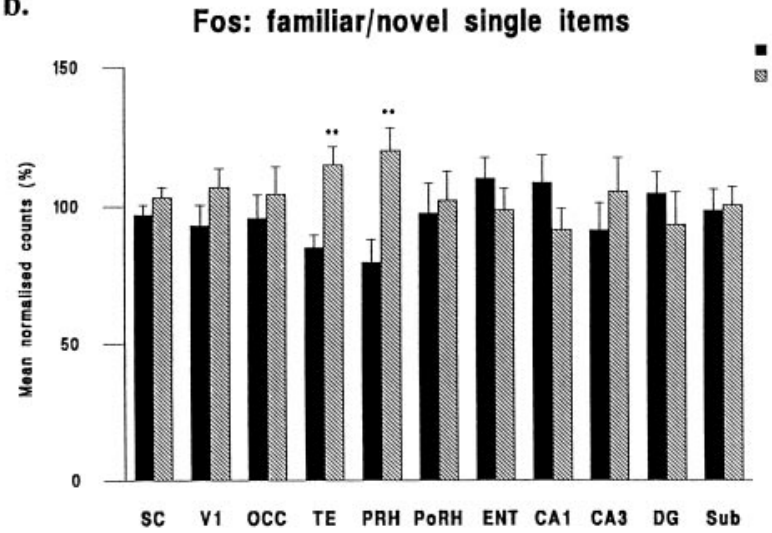

c.

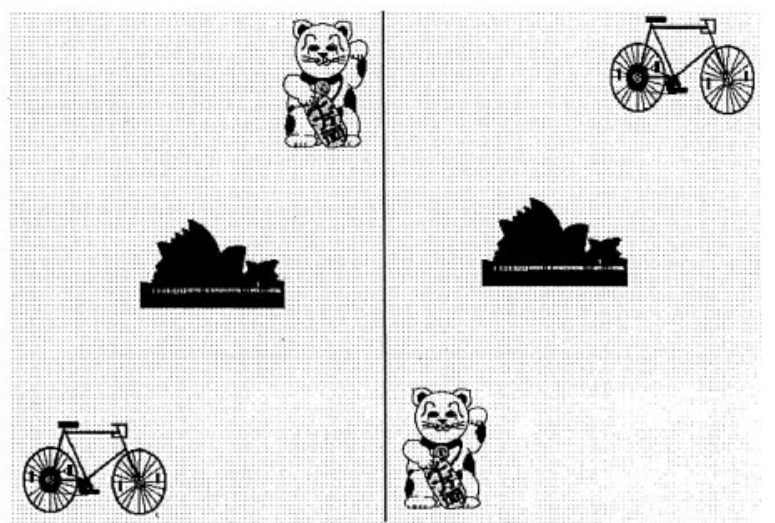

d. Fos: familiar/novel arrangements of items

- familiar novel

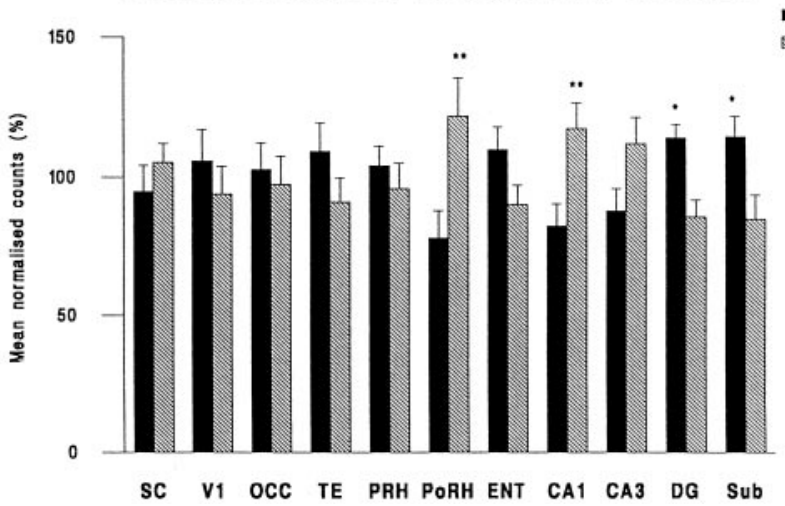

Figure 3. Example pictures and normalized counts in the brain regions sampled for novel and familiar individual items and arrangements of items. $a$, Two example pictures of single items (items were colored but are illustrated in monochrome). $b$, Normalized counts of Fos-stained nuclei in the sampled brain regions for novel and familiar pictures of single items. An ANOVA of the normalized counts revealed a significant interaction between area and novelty/familiarity $\left(F_{(10,360)}=2.18 ; p<0.02\right)$. Further analysis revealed that the mean counts were higher for novel than for familiar objects in areas TE and PRH (each $p<0.01$ ), but no other differences reached significance. $c$, Two example pictures of arrangements of items: note the different arrangements of the same items. $d$, Normalized counts of Fos-stained nuclei in the sampled brain regions for novel and familiar pictures of arrangements of items. An ANOVA of the normalized counts revealed a significant interaction between area and novelty/familiarity $\left(F_{(10,306)}=5.69 ; p<0.001\right)$. Further analysis revealed that the mean counts were higher for novel than for familiar arrangements in areas PoRH and CA1 (each $p<0.01$ ) and higher for familiar than for novel arrangements in areas DG and Sub (each $p<0.05$ ). No other differences reached significance. Although the size of each individual stimulus was larger in experiment 1 than experiment 2, the percentage differences in activation for novel and familiar stimuli are comparable to those found using objects that subtend approximately half the visual angle of these stimuli (Zhu et al., 1996). Accordingly, the size of the individual items is not critical to the results obtained over the range of sizes explored. Novel versus familiar differences: ${ }^{*} p<0.05 ; * * p<0.01$. See Figure 2 for abbreviations.

\section{RESULTS}

In the first experiment, the rats saw pictures of individual items (Fig. 3a). Counts of neuronal nuclei stained for Fos were significantly higher for novel than for familiar pictures in area TE of the temporal lobe (TE) and perirhinal cortex (PRH); see Figures 2, 3b, and 4 (for statistical details, see the legend of Fig. $3 b$; also see Fig. 4 for example photomicrographs of Fos staining). No significant difference was found in any other area examined, including within the hippocampal formation, i.e., hippocampal subfields CA1, CA3, the dentate gyrus (DG), and the subiculum (Sub), or postrhinal cortex (PoRH). Counts for all areas in both experiments are given in Table 1. Counts in the hippocampal formation were low (mean $\pm \mathrm{SEM}=6.4 \pm 1.04 \mathrm{~mm}^{-2}$ ), i.e., relatively few hippocampal neurons were activated by these pictures of individual items, compared with the higher counts in PRH $\left(30.3 \pm 6.2 \mathrm{~mm}^{-2}\right)$, PoRH $\left(24.0 \pm 7.3 \mathrm{~mm}^{-2}\right)$, and TE $\left(28.7 \pm 5.9 \mathrm{~mm}^{-2}\right)$.

In the second experiment, the rats saw pictures of spatial arrangements of individual items (Fig. 3c). Activation was compared for novel and familiar arrangements of the same familiar items. Counts of neuronal nuclei stained for Fos were significantly higher for novel than for familiar arrangements in PoRH and CA1, but significantly lower in the DG and Sub; see Figure $3 d$ and legend for details; also see Figure 5 for examples of photomicrographs of Fos staining. No significant difference was found in any other area examined, including TE and PRH.

Counts in the hippocampal formation were higher for pictures of arrangements of items $\left(20.8 \pm 2.9 \mathrm{~mm}^{-2}\right)$ than for individual items. Thus, the ratio of the counts in the hippocampal formation to that in occipital visual association cortex (OCC) was significantly higher (2.5 times higher) for arrangements of items than for individual items (ratio, $0.61 \pm 0.06$ compared with $0.25 \pm$ 0.04 ; independent $t$ test; $p<0.01$ ), the counts in occipital cortex being approximately the same for both types of pictures (34.1 \pm 12.2 for arrangements and $31.0 \pm 6.4$ for individual items). Mean counts in other areas did not vary significantly between the two 


\section{Novel}

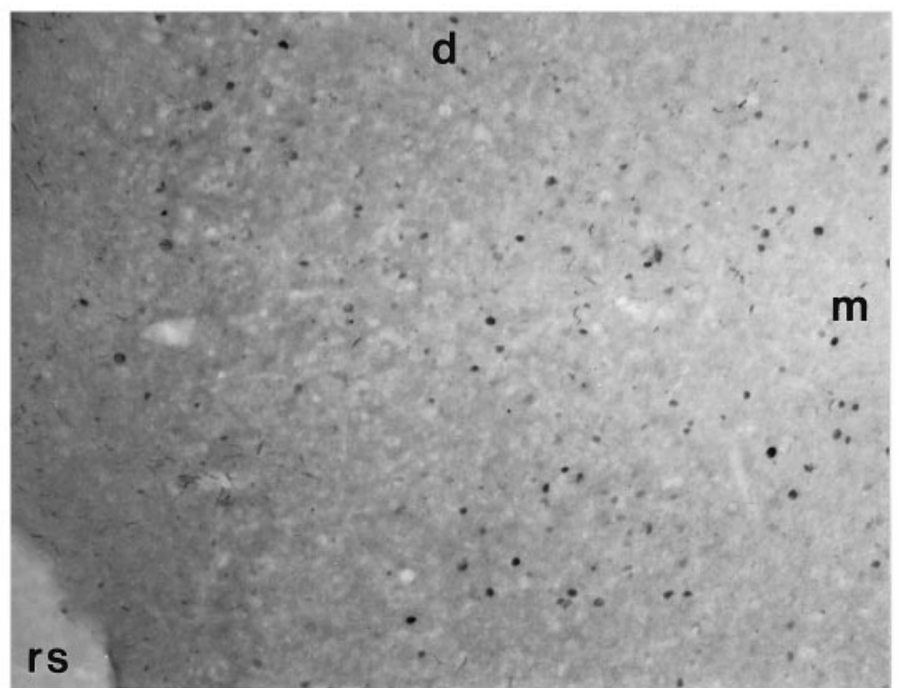

Familiar

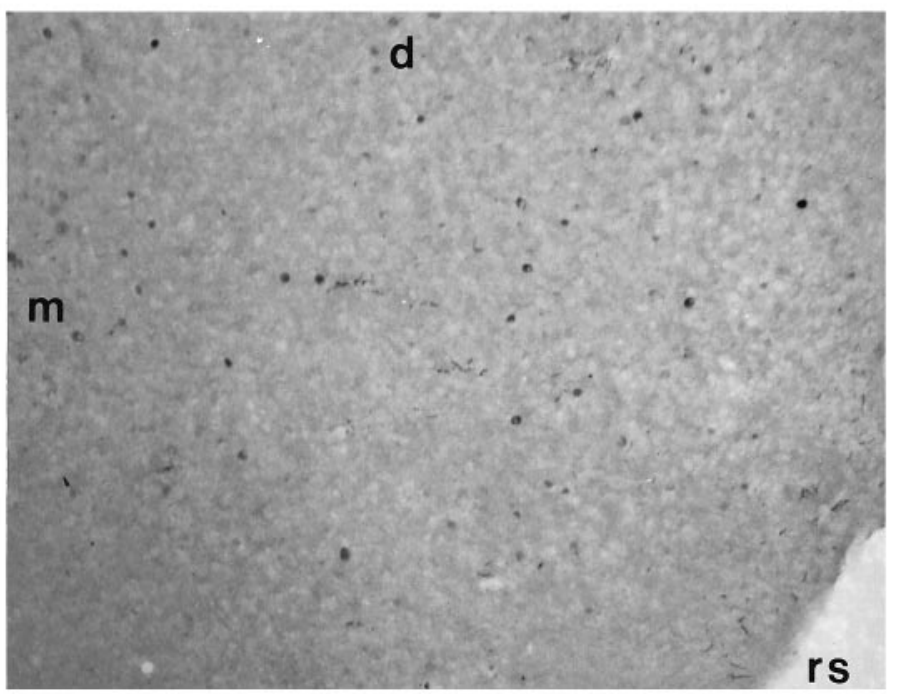

Figure 4. Activated neurons (Fos-stained nuclei) in perirhinal cortex as a result of viewing individual items: novel (left) or familiar (right). Note the greater number of stained nuclei on the left (novel). Coronal sections (5.20 behind bregma); magnification, $83 \times ; d$, dorsal; $m$, medial; rs, rhinal sulcus.

\begin{tabular}{|c|c|c|}
\hline Area & Experiment 1 & Experiment 2 \\
\hline $\mathrm{SC}$ & $74.3 \pm 13.7$ & $72.6 \pm 15.4$ \\
\hline V1 & $26.4 \pm 6.7$ & $18.1 \pm 4.1$ \\
\hline OCC & $31.0 \pm 6.4$ & $34.1 \pm 12.2$ \\
\hline TE & $28.7 \pm 5.9$ & $33.0 \pm 4.4$ \\
\hline $\mathrm{PRH}$ & $30.3 \pm 6.2$ & $79.5 \pm 15.9$ \\
\hline PoRH & $24.0 \pm 7.3$ & $37.3 \pm 9.4$ \\
\hline ENT & $18.9 \pm 4.3$ & $32.6 \pm 8.3$ \\
\hline CA1 & $3.3 \pm 0.5$ & $13.0 \pm 4.4$ \\
\hline CA3 & $4.4 \pm 0.5$ & $14.4 \pm 3.3$ \\
\hline DG & $10.0 \pm 2.9$ & $36.4 \pm 3.0$ \\
\hline Sub & $7.9 \pm 1.1$ & $17.1 \pm 3.3$ \\
\hline
\end{tabular}

experiments. Hence, the change in counts was regionally specific. Many of the stained nuclei in the dentate gyrus and the subfield CA1 of the hippocampus were located in the principal cell body layers (stratum granulosum and stratum pyramidale), but there were some stained nuclei located outside these layers i.e., the activated neurons included putative interneurons (Fig. 5).

\section{DISCUSSION}

The results establish that pictures of novel arrangements of familiar items produce significantly greater activation than familiar arrangements of these items in postrhinal cortex and subfield CA1 of the hippocampus, but significantly less activation in the dentate gyrus and subiculum; perirhinal cortex and area TE are not differentially activated. In contrast, perirhinal cortex and area TE of the temporal lobe are activated significantly more by pictures of novel than of familiar individual objects, but the hippocampus is not differentially activated.

The results of the first experiment are in agreement with previous findings using Fos imaging for either between- or withinrat comparisons but using individual three-dimensional objects as opposed to computer-displayed pictures (Zhu et al., 1995b, 1996). The consistency of the Fos difference in perirhinal cortex and area TE for novel and familiar individual items across the three experiments firmly establishes the robustness of the findings. Moreover, the Fos results are in accord with results of electrophysiological recordings and ablation studies in the primate and in the rat (Brown et al., 1987; Horel et al., 1987; Gaffan and Murray, 1992; Mumby and Pinel, 1994; Wiig and Bilkey, 1995; Zhu et al., 1995a; Ennaceur et al., 1996; Murray, 1996; Suzuki, 1996; Aggleton et al., 1997; Brown and Xiang, 1998; Xiang and Brown, 1998; Aggleton and Brown, 1999). In particular, novel objects or pictures of objects evoke greater neuronal responses than do familiar objects in area TE and PRH, and such differential responses are uncommon in the hippocampus (Riches et al., 1991; Rolls et al., 1993; Xiang and Brown, 1998). Although Fos staining was used in these experiments merely as a marker of neuronal activation, it is worth noting that Fos also provides a marker for long-term depression in the cerebellum (Nakazawa et al., 1993), another process in which neuronal responsiveness is reduced as a result of experience.

Neuronal responses to novel and familiar arrangements of items, the stimuli used in the second experiment, have yet to be explored in recording experiments. Although novel pictures resulted in the activation of more perirhinal neurons than did familiar pictures when the pictures were of single items, no differential activation was found when the pictures were of arrangements of items. This contrast is explicable because all the individual items in the arrangements were equally familiar. In contrast, a significant difference was observed in PoRH for novel and familiar arrangements, although not for novel and familiar single items. This finding is in accord with the idea that postrhinal cortex in the rat is concerned with processing spatial information and is consistent with the suggestion that rat postrhinal cortex has homologies with areas TF and TH of the parahippocampal cortex in the monkey (Burwell et al., 1995). Regions that process spatial information project heavily to this parahippocampal cortex in the monkey (Burwell et al., 1995). 
Novel
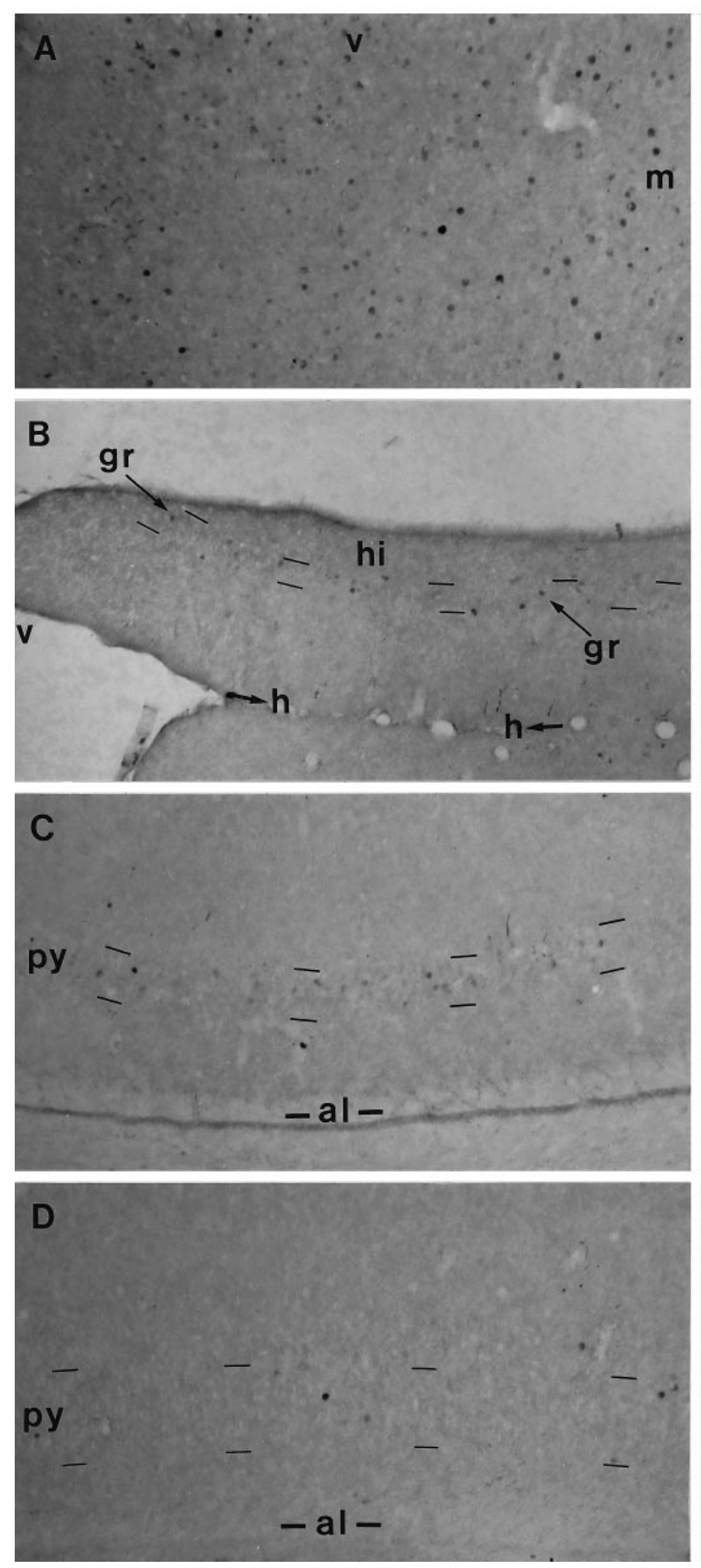

\section{Familiar}
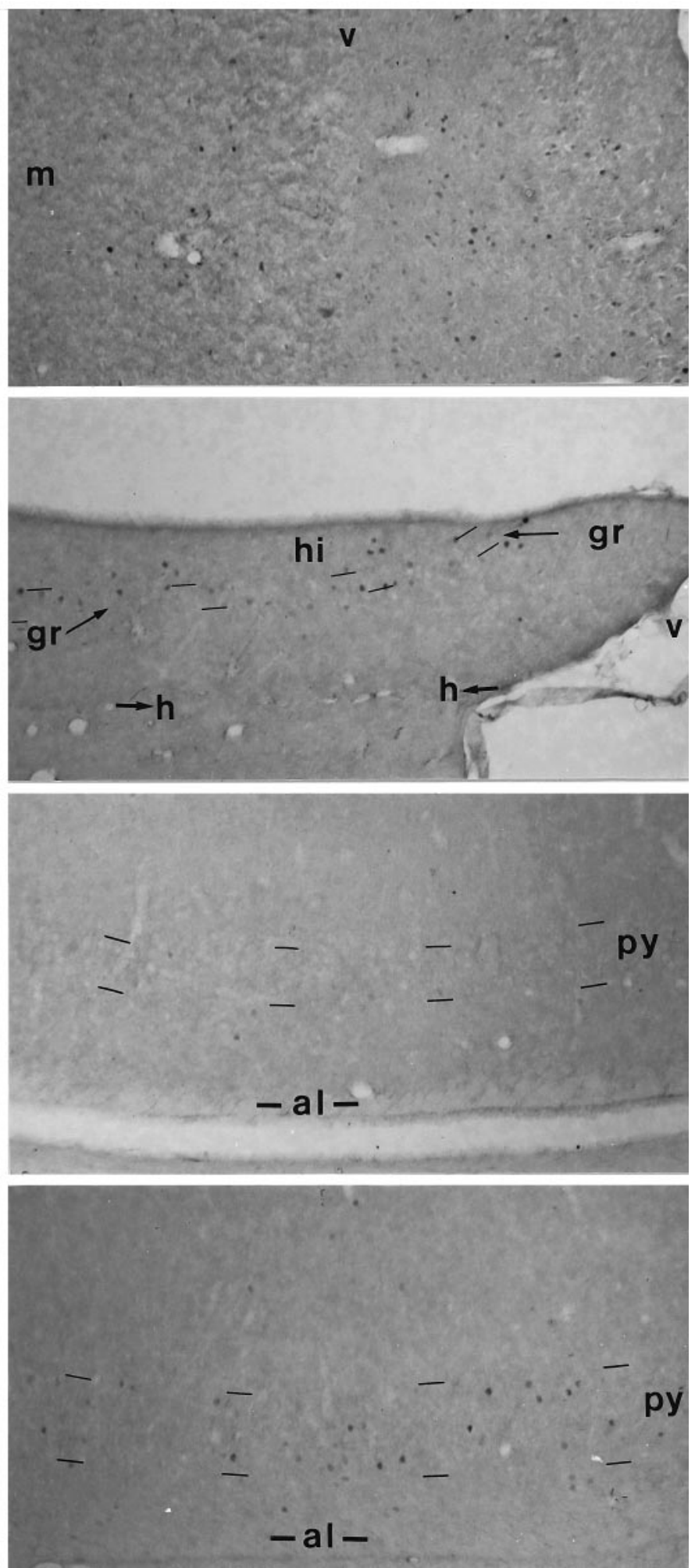

Figure 5. Activated neurons (Fos-stained nuclei) as a result of viewing arrangements of items: novel (left) or familiar (right) in $(A)$ postrhinal cortex (7.80), $(B)$ dentate gyrus (5.20), $(C)$ CA1 (5.20), and $(D)$ subiculum (5.20). Note that certain stained nuclei lie outside the principal cell layers and hence are probably interneurons. Coronal sections at given distances behind bregma (see also Fig. 2); magnification, $83 \times$; $a l$, alveus; $g r$, stratum granulosum; $h$, hippocampal fissure; $m$, medial; $p y$, stratum pyramidale; $v$, ventral. 
These results help to resolve an apparent conflict over the contribution of the hippocampus to recognition memory by establishing that its involvement is dependent on the form of what has to be recognized. Thus, they add to evidence that the hippocampus is not critical for recognition memory judgments of the relative familiarity of individual items (Aggleton and Shaw, 1996; Ennaceur et al., 1996, 1997; Murray, 1996; Murray and Mishkin, 1998; but see Alvarez et al., 1995). However, they establish that the hippocampus is importantly involved in discriminating the relative familiarity of spatial arrangements of items. Memory for such spatial arrangements is an important aspect of episodic memory in which whether a particular scene, i.e., a particular arrangement of items, has been encountered before needs to be determined, especially if many of the individual items have been seen before but their particular configuration is novel. Thus, recognition memory tasks that can be readily solved by discriminating the relative familiarity of individual items, whether in isolation or as part of a scene, would not require the hippocampus, in agreement with previous animal lesion studies. However, the hippocampus would provide an important contribution to recognition memory in more complex, everyday life situations in which the memory is for an environment or recognition is assisted by recollection of the original episode (Brown, 1990; Gaffan, 1991, 1994; Ennaceur et al., 1996, 1997; Gaffan and Parker, 1996; Murray, 1996; Murray and Mishkin, 1998; Nadel and Moscovitch, 1997; Brown and Xiang, 1998; Aggleton and Brown, 1999). In support of this view, previous evidence, both from recording and from recent functional imaging studies, has indicated the importance of spatial and other associational information to the responsiveness of hippocampal neurons (O'Keefe and Nadel, 1978; Eichenbaum et al., 1994, 1996; Wiener, 1996; Maguire, 1997). It is worth noting that for human subjects performing memory tasks it would seem normal for the subjects to make spatial and other associative links (including attaching verbal labels) involving the presented material whatever the nature of the material.

Importantly, our findings demonstrate that one subregion of the hippocampal formation can be activated in the opposite way to other subregions: subfield CA1 showed greater activation for novel than familiar arrangements, whereas the dentate gyrus and subiculum showed more activation for familiar than for novel arrangements. These opposing changes could be explained by the influence of inhibitory interneurons as such information passes from one subregion to the next through the hippocampal formation. Consistent with this idea is the discovery of stained nuclei outside the principal cell layers, suggesting that at least some of the activated neurons are indeed interneurons (Fig. 5B). In agreement with this finding of opposite changes in different subregions of the hippocampal formation is a recent brief report indicating that neurons in subfield CA1 respond more vigorously in a novel environment than in a familiar environment, whereas neurons in the dentate gyrus do the converse (Nitz et al., 1997). Different c-fos mRNA levels in hippocampal subfield CA1 compared with CA3 and the dentate gyrus have also been observed during exploration and odor discrimination learning (Hess et al., 1995a,b). A further possibility is that the differential activation is related to differences in processing between the dorsal and ventral hippocampal formation: lesion and electrophysiological studies have indicated that the dorsal hippocampal formation is more important than the ventral for spatial learning (Jung et al., 1994; Moser et al., 1995). However, although in the present study the sampled part of CA1 was dorsal, whereas that of the dentate gyrus was ventral, the ventral as well as the dorsal sample were differ- entially activated (the effect being opposite in the two areas). Should such differential hippocampal activation occur in the human brain, it will be important that human functional imaging studies have a resolution sufficient to differentiate between hippocampal subregions, because a global hippocampal average may not show a net difference. Accordingly, such subregion differences provide yet another possible reason for failures of functional imaging to detect significant hippocampal changes during memory tasks (Nyberg et al., 1996; Maguire, 1997; Tulving and Markowitsch, 1997).

The findings of the present study provide a clear demonstration of the different contributions to recognition memory of the hippocampal formation and perirhinal cortex. The role of perirhinal cortex is in judgment of the relative familiarity of an individual item. The role of the hippocampus is in judgment of the relative familiarity of an arrangement of items. This latter capacity is necessary for episodic memory for complex events involving relationships among many individual items, and our findings establish an essential function for the hippocampus in such memory.

\section{REFERENCES}

Aggleton JP, Brown MW (1999) Episodic memory, amnesia and the hippocampal-anterior thalamic axis. Behav Brain Sci, in press.

Aggleton JP, Shaw C (1996) Amnesia and recognition memory: a reanalysis of psychometric data. Neuropsychologia 34:51-62.

Aggleton JP, Keen S, Warburton EC, Bussey TJ (1997) Extensive cytotoxic lesions involving both the rhinal cortices and area TE impair recognition but spare spatial alternation in the rat. Brain Res Bull 43:279-287.

Alvarez P, Zola-Morgan S, Squire LR (1995) Damage limited to the hippocampal region produces long-lasting memory impairment in monkeys. J Neurosci 15:3796-3807.

Brennan PA, Hancock D, Keverne EB (1992) The expression of the immediate-early genes c-fos, egr- 1 and c-jun in the accessory olfactory bulb during the formation of an olfactory memory in mice. Neuroscience 101:274-280.

Brown MW (1990) Why does the cortex have a hippocampus? In: Learning and computational neuroscience: foundations of adaptive networks (Gabriel M, Moore J, eds), pp 233-282. New York: MIT.

Brown MW (1996) Neuronal responses and recognition memory. Semin Neurosci 8:23-32.

Brown MW, Wilson FAW, Riches IP (1987) Neuronal evidence that inferomedial temporal cortex is more important than hippocampus in certain processes underlying recognition memory. Brain Res 409:158-162.

Brown MW, Xiang J-Z (1998) Recognition memory: neuronal substrates of the judgment of prior occurrence. Prog Neurobiol 55:149-189.

Burwell RD, Witter MP, Amaral DG (1995) Perirhinal and postrhinal cortices of the rat: a review of the neuroanatomical literature and comparison with findings from the monkey brain. Hippocampus 5:390-408

Dragunow M (1996) A role for immediate-early transcription factors in learning and memory. Behav Genet 26:293-299.

Eichenbaum H, Otto T, Cohen NJ (1994) Two functional components of the hippocampal memory system. Behav Brain Sci 17:449-518.

Eichenbaum H, Schoenbaum G, Young B, Bunsey M (1996) Functional organization of the hippocampal memory system. Proc Natl Acad Sci USA 93:13500-13507.

Ennaceur A, Neave N, Aggleton JP (1996) Neurotoxic lesions of the perirhinal cortex do not mimic the behavioural effects of fornix transection in the rat. Behav Brain Res 80:9-25.

Ennaceur A, Neave N, Aggleton JP (1997) Spontaneous object recognition and object location memory in rats: the effects of lesions in the cingulate cortices, the medial prefrontal cortex, the cingulum bundle and the fornix. Exp Brain Res 113:509-519.

Gaffan D (1991) Spatial organisation of episodic memory. Hippocampus 1:262-264. 
Gaffan D (1994) Scene-specific memory for objects: a model of episodic memory impairment in monkeys with fornix transection. J Cognit Neurosci 6:4:305-320.

Gaffan D, Murray EA (1992) Monkeys (Macaca fascicularis) with rhinal cortex ablations succeed in object discrimination learning despite $24-\mathrm{hr}$ intertrial intervals and fail at matching to sample despite double sample presentations. Behav Neurosci 106:30-38.

Gaffan D, Parker A (1996) Interaction of perirhinal cortex with the fornix-fimbria: memory for objects and "object-in-place" memory. J Neurosci 16:5864-5869.

Hess US, Lynch G, Gall CM (1995a) Changes in c-fos mRNA expression in rat brain during odor discrimination learning: differential involvement of hippocampal subfields CA1 and CA3. J Neurosci 15:4786-4795.

Hess US, Lynch G, Gall CM (1995b) Regional patterns of c-fos mRNA expression in rat hippocampus following exploration of a novel environment versus performance of a well-learned discrimination. J Neurosci 15:7796-7809.

Horel JA, Pytkojoiner DE, Voytko M, Salsbury K (1987) The performance of visual tasks while segments of the inferotemporal cortex are suppressed by cold. Behav Brain Res 23:29-42.

Jung MW, Wiener SI, McNaughton BL (1994) Comparison of spatial firing characteristics of units in dorsal and ventral hippocampus of the rat. J Neurosci 14:7347-56.

Larea LS (1997) Glutamate receptors and gene induction: signalling from receptor to nucleus. Cell Signal 9:219-226.

Maguire EA (1997) The cerebral representation of space: insights from functional imaging data. Trends Cognit Sci 1:62-68.

McCabe B, Horn G (1994) Learning-related changes in Fos-like immunoreactivity in the chick forebrain after imprinting. Proc Natl Acad Sci USA 91:11417-11421.

Morgan JI, Curran T (1991) Stimulus-transcription coupling in the nervous system: involvement of the inducible proto-oncogenes fos and jun. Annu Rev Neurosci 14:421-451.

Moser MB, Moser EI, Forrest E, Andersen P, Morris RGM (1995) Spatial learning with a minislab in the dorsal hippocampus. Proc Natl Acad Sci USA 92:9697-9701.

Mumby DG, Pinel JPJ (1994) Rhinal cortex lesions and object recognition in rats. Behav Neurosci 108:11-18.

Murray EA (1996) What have ablation studies told us about the neural substrates of stimulus memory? Semin Neurosci 8:13-22.

Murray EA, Mishkin M (1998) Object recognition and location memory in monkeys with excitotoxic lesions of the amygdala and hippocampus. J Neurosci 18:6568-6582.

Nadel L, Moscovitch M (1997) Memory consolidation, retrograde amnesia and the hippocampal complex. Curr Opin Neurobiol 7:217-227.

Nakazawa K, Karchot L, Nakabeppu Y, Yamamori T (1993) The conjunctive stimuli that cause long-term desensitisation also predominantly induce c-fos and jun-B in cerebellar Purkinje cells. NeuroReport 4:1275-1278.

Nitz DA, Pauer M, McNaughton BL (1997) Divergent modulation of CA1 and DG interneurons by spatial novelty. Soc Neurosci Abstr 23:196.4.

Nyberg L, McIntosh AR, Cabeza R, Habib R, Houles S, Tulving E (1996) General and specific brain regions involved in encoding and retrieval of events: what, where, and when. Proc Natl Acad Sci USA 93:11280-11285.

O'Keefe J, Nadel L (1978) The hippocampus as a cognitive map. Oxford: Oxford UP.

Paxinos G, Watson C (1986) The rat brain in stereotaxic co-ordinates. London: Academic.

Riches IP, Wilson FAW, Brown MW (1991) The effects of visual stimulation and memory on neurons of the hippocampal formation and the neighboring parahippocampal gyrus and inferior temporal cortex of the primate. J Neurosci 11:1763-1779.

Rolls ET, Cahusac PMB, Feigenbaum JD, Miyashita Y (1993) Responses of single neurons in the hippocampus of the macaque related to recognition memory. Exp Brain Res 93:299-306.

Sefton AJ, Dreher B (1995) Visual system. In: The rat nervous system, Vol 1, Forebrain and midbrain (Paxinos G, ed), pp 833-898. New York: Academic.

Sheng M, Greenberg ME (1990) The regulation and function of c-fos and other immediate early genes in the nervous system. Neuron 4:477-485.

Suzuki WA (1996) The anatomy, physiology and functions of the perirhinal cortex. Curr Opin Neurobiol 6:179-186.

Tulving E, Markowitsch HJ (1997) Memory beyond the hippocampus. Curr Opin Neurobiol 7:209-216.

Wiener SI (1996) Spatial, behavioral and sensory correlates of hippocampal CA1 complex spike cell activity: implications for information processing functions. Prog Neurobiol 49:335-361.

Wiig KA, Bilkey DK (1995) Lesions of rat perirhinal cortex exacerbate the memory deficit observed following damage to the fimbria-fornix. Behav Neurosci 109:620-630.

Xiang J-Z, Brown MW (1998) Neuronal encoding of novelty and familiarity in the anterior medial temporal lobe. Neuropharmacology 37:657-676.

Zhu XO, Brown MW, Aggleton JP (1995a) Neuronal signalling of information important to visual recognition memory in rat rhinal and neighbouring cortices. Eur J Neurosci 7:753-765.

Zhu XO, Brown MW, McCabe BJ, Aggleton JP (1995b) Effects of novelty or familiarity of visual stimuli on the expression of the immediate early gene c-fos in rat brain. Neuroscience 69:821-829.

Zhu XO, McCabe BJ, Aggleton JP, Brown MW (1996) Mapping visual recognition memory through expression of the immediate early gene c-fos. NeuroReport 7:1871-1875. 